\title{
Etude de la dynamique des électrons à deux dimensions dans les hétérojonctions
}

\section{J. Zimmermann et Wu Yen}

Centre Hyperfréquences et Semiconducteurs U.A. au C.N.R.S. n $^{\circ} 287$, Université de Lille I, 59655 Villeneuve d'Ascq Cedex, France

(Reçu le 23 janvier 1987, révisé le 21 mai 1987, accepté le 18 juin 1987)

\begin{abstract}
Résumé. - L'étude détaillée de la dynamique électronique à deux dimensions est devenue un besoin avec l'avènement des composants utilisant les hétérojonctions comme, par exemple le HEMT. Nous décrivons en détail un modèle de type Monte Carlo qui permet une étude aisée des phénomènes de transport en champ électrique fort. Entre autres, nous proposons un modèle prenant en compte le couplage entre un système d'électrons à deux dimensions et un système d'électrons à trois dimensions coexistant dans le même matériau. Nous calculons ainsi la vitesse de dérive électronique en fonction des polarisations appliquées au système. Une première approche du phénomène de diffusion.est également effectuée.
\end{abstract}

\begin{abstract}
With the recent advent of devices using heterojunctions, like HEMTs, there exists now a growing interest in the physical study of two-dimensional electron dynamics. In this paper, we detail a Monte Carlo model which allows one to easily study transport phenomena at high electric field. We also present a model for the coupling between a two-dimensional electron system and a three-dimensional electron system embedded in the same material. One then computes the electron drift velocity as function of bias fields applied to the system. A preliminary approach of diffusion is also carried out.
\end{abstract}

\section{Introduction.}

Dans cet article nous nous proposons de décrire un modèle de la dynamique d'électrons à deux dimensions qui tient compte des effets très particuliers qui régissent le transport de charge dans les nouveaux composants à hétérojonctions tel que les HEMTs [14]. Le modèle est ensuite étudié grâce à une méthode de Monte Carlo.

La structure du HEMT est représentée sur la figure 1a. Il est constitué d'un empilement de couches épitaxiales qui sont (depuis le substrat semiisolant) : une couche tampon AsGa non dopée, une fine couche AlGaAs non dopée (appelée «spacer »), une couche AlGaAs fortement dopée de type $\mathrm{n}\left(\sim 10^{18} \mathrm{~cm}^{-3}\right)$, une couche AsGa fortement dopée de type $n$ (pour les contacts de source et de drain) qui est recessée pour le dépôt d'une grille métallique directement sur le AlGaAs dopé. La barrière existant au niveau du contact de grille, due à la barrière Schottky à laquelle s'ajoute la tension de polarisation de grille $V_{\mathrm{g}}$, permet de dépléter la couche dopée de ses électrons qui viennent s'accumuler (par effet de charge d'espace) à l'interface $\mathrm{AlGaAs}-\mathrm{AsGa}$, où

REVUE DE PHYSIQUE APPLIQUÉE. - T. 22, N` 11, NOVEMBRE 1987 existe la barrière de potentiel due à l'hétérojonction empêchant les électrons de retourner dans la couche AlGaAs. La bande de conduction va donc se courber vers les énergies plus faibles pour permettre à une forte densité d'électrons de demeurer au voisinage de l'interface. Il y a formation d'un puits de potentiel dont la largeur (100 à $200 \AA ̊)$ permet à des effets quantiques d'entrer en jeu. Ceci se traduit par la formation d'une série de sous-bandes d'énergie pour les électrons confinés dans le puits. La situation ainsi obtenue est résumée dans la figure $1 \mathrm{~b}$. Ces sousbandes n'existent cependant que pour l'énergie correspondant au mouvement au travers du puits (direction $z$ de la Fig. 1b). Dans le modèle, l'électron sera considéré comme quasi libre dans les deux autres directions $x$ et $y$.

Le canal conducteur du HEMT est donc constitué de ces électrons à deux dimensions dont on commande la densité par la tension de grille $V_{\mathrm{g}}$. D'après nombre d'auteurs, ce gaz électronique à deux dimensions est doué de bonnes propriétés de transport aussi bien pour la mobilité que pour le bruit [5]. Une des raisons principales est la possibilité de séparer les électrons de leurs impuretés mères 


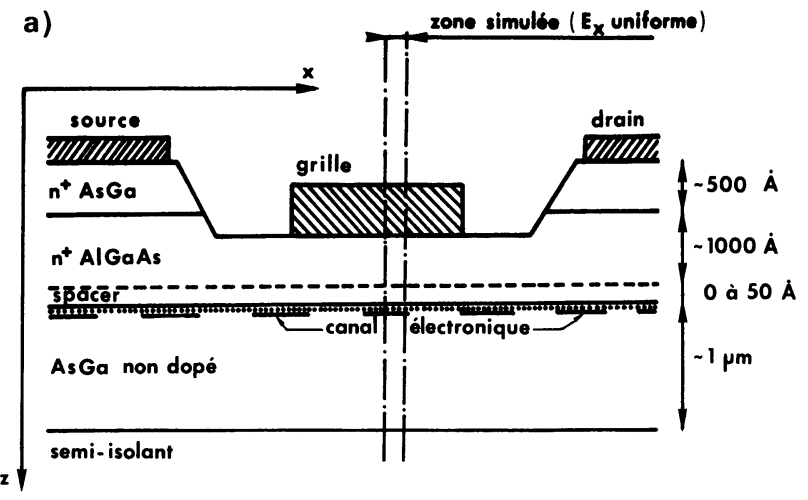

b)

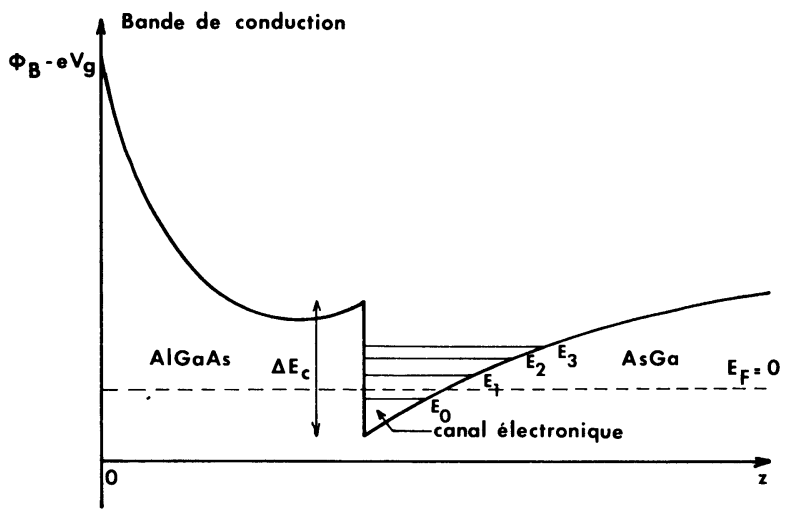

Fig. 1. - Constitution générale du HEMT (cadre du haut). La bande de conduction présentera une série de sous-bandes $E_{n}$ (au lieu du continuum $E_{z}=\hbar^{2} /\left(2 m^{*}\right)$ $k_{z}^{2}$ lorsque la largeur du puits aux environs du niveau de Fermi $E_{\mathrm{F}}$ n'est pas beaucoup plus grande que la longueur d'onde de Broglie des électrons dans la direction $z$.

[Usual constitution of a HEMT (at the top). The conduction band (bottom) will present a subband energy scale rather than a continuum whenever the well-width near the Fermi level is in the order of magnitude of the electron thermal wavelength.]

grâce à l'existence du «spacer » qui permet de réduire notablement l'effet à distance du potentiel ionique coulombien agissant sur les électrons. Par exemple, des mobilités électroniques de $200000 \mathrm{~cm}^{2} / \mathrm{V} / \mathrm{s}$ à $77 \mathrm{~K}$ et 9200 à $300 \mathrm{~K}$ ont été mesurées récemment [6]. L'intérêt du composant par rapport au transistor MES classique, est de pouvoir obtenir des densités électroniques très fortes (en densité volumique elles peuvent atteindre $2 \times 10^{18} \mathrm{~cm}^{-3}$ ) dans un matériau très pur et donc de profiter pleinement d'une mobilité élevée nécessaire pour atteindre des temps de réponse les plus courts possibles [2] dans les applications en circuits intégrés logiques, ou les fréquences de coupure les plus grandes possibles dans les applications en circuits intégrés hyperfréquences.

Notre propos dans la suite de cet article sera donc d'examiner cette nouvelle dynamique qui, par maints aspects, se rapproche de ce qui se passe dans les couches d'inversion des transistors MOS. Nous nous attachons à étudier les phénomènes de porteurs chauds qui se manifestent lorsqu'on applique un champ accélérateur élevé parallèlement à l'hétérojonction tel qu'il résulte de la polarisation sourcedrain du HEMT. Nous déterminons la vitesse de dérive et dans une étape ultérieure nous étudions le phénomène de diffusion. Ce dernier, qui n'a encore jamais été abordé, est important en relation avec les propriétés de bruit de fond qui spécifient les limites ultimes de fonctionnement de ces composants. Dans cette étude, le champ accélérateur (selon $x$ ) est considéré comme uniforme. Nous étudions donc ce qui se passe en fait à l'intérieur d'une tranche du composant découpée perpendiculairement au plan de la grille.

\section{Le modèle.}

2.1 ASPECTS QUALITATIFS. - Un électron que nous supposerons placé sur une des sous-bandes du puits formé à l'hétérojonction est soumis au champ accélérateur et voit son énergie varier au cours du temps. Soumis également aux diverses collisions susceptibles de se produire, l'électron, spécifié par un indice de sous-bandes et un vecteur d'onde à deux composantes $\mathbf{k}_{\|}=\left(k_{x}, k_{y}\right)$, pourra passer d'une sous-bande à une autre en émettant ou en absorbant l'énergie de phonons. Parvenu à une énergie suffisamment élevée il aura la possibilité de passer dans une vallée satellite ( $\mathrm{L}$ ou $\mathrm{X}$ ) dans $\mathrm{AsGa}$ ou dans AlGaAs (sous certaines conditions). Son mouvement est alors supposé être quasi tridimensionnel et analogue à ce qui existe dans le même matériau en volume. Ceci autorise ainsi le passage ultérieur vers une vallée $\Gamma$ à trois dimensions cette fois, avec la condition que cette transition ne pourra se faire qu'en un point où l'électron est suffisamment loin de l'hétérojonction. Cette situation est résumée dans la figure 2. Dans le modèle, cette condition est exprimée simplement en termes de fonction d'onde et de largeur effective de sous-bande. $\mathrm{Si}$, au contraire, l'électron situé dans la vallée satellite se trouve au niveau du puits, seul un transfert vers une des sousbandes de la vallée $\Gamma$ à deux dimensions sera possible. On voit donc que, globalement, l'histoire de l'électron sera décomposée en phases successives durant lesquelles le mouvement sera soit purement bidimensionnel soit purement tridimensionnel. Dans le cas du champ faible, près de l'équilibre thermique, l'électron restera piégé dans la vallée $\Gamma$ à deux dimensions et son mouvement restera purement bidimensionnel. On voit par ailleurs qu'un couplage direct entre la vallée $\Gamma$ à deux dimensions et la vallée $\Gamma$ à trois dimensions n'est pas possible dans la mesure où ces deux vallées ne coexistent pas dans la même région de l'espace. Pour résumer, il est 


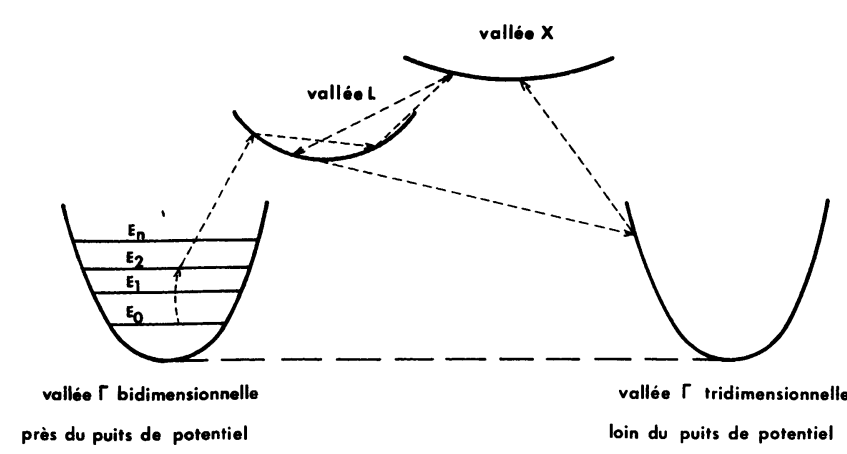

Fig. 2. - Cette figure représente une trajectoire typique d'un électron qui étant défini à deux dimensions dans la vallée $\Gamma$ au départ passe dans une des vallées satellites où son mouvement devient tridimensionnel au cours d'une collision suivi d'un retour éventuel dans la vallée $\Gamma$ à trois dimensions. Le retour vers la vallée $\Gamma$ à deux dimensions ne peut s'effectuer que par l'intermédiaire d'une vallée satellite et à condition que l'électron se trouve réellement au-dessus du puits de potentiel comme précisé dans le texte. Dans le cas du champ faible, l'électron ne peut pas sortir de la vallée $\Gamma$ à deux dimensions et son mouvement reste purement bidimensionnel.

[Represents a typical trajectory of a hot electron between two-dimensional and three-dimensional states.]

important de se souvenir du rôle primordial que joue la position $z$ de l'électron dans l'espace pour la détermination de la nature de son mouvement.

Considérer l'électron comme quasi tridimensionnel lorsqu'il évolue dans les vallées satellites est raisonnable dans la mesure où d'une part la séparation des sous-bandes dans celle-ci est, pour une densité surfacique $n_{\mathrm{s}}$ donnée, nettement plus faible que dans la vallée $\Gamma$, ce qui est dû à la masse plus élevée dans les vallées $L$ et $X$. D'autre part, du moins au-dessous du champ de seuil, ces dernières sont vides d'électrons. Lorsqu'elles ne le sont plus, du fait du transfert de la vallée centrale $\Gamma$ vers cellesci et à champ assez élevé, la distribution thermique des électrons aura tendance à recouvrir un assez grand nombre de sous-bandes d'où il résulte que le caractère bidimensionnel du mouvement électronique dans les vallées satellites est fortement diminué.

Pour mener à bien un modèle dynamique utilisable avec une méthode de Monte Carlo, deux ensembles de données sont nécessaires. D'une part, il faut connaître la forme des bandes d'énergie où vont évoluer les particules, d'autre part, il faut connaître les expressions de toutes les probabilités de collisions que ces particules sont susceptibles de subir durant leur mouvement. Nous abordons ces deux points dans les deux paragraphes qui suivent.

2.2 LES BANDES D'ÉNERGIE DANS LES HÉTÉROJONCTIONS. - L'énergie suivant la direction $z$ étant quantifiée, l'expression de la bande de conduction aura la forme :

$$
E(\mathbf{k})=E_{n}+E_{\|}=E_{n}+\left(\hbar^{2} / 2 m^{*}\right)\left(k_{x}^{2}+k_{y}^{2}\right),
$$

où $n$ est un indice de sous-bandes et la particule ellemême sera décrite par une fonction d'onde de la forme :

$$
\psi_{n, \mathbf{k}_{1}}=\varphi_{n}(z) \cdot \exp \left[i\left(k_{x} x+k_{y} y\right)\right],
$$

où $\varphi_{n}(z)$ est la fonction d'onde enveloppe de l'électron dans la direction $z$. La forme de $\varphi_{n}$ et les valeurs de $E_{n}$ dépendent bien sûr de la forme du puits de potentiel. Au début de notre étude nous avions envisagé le cas d'un puits rectangulaire profond constitué par une double hétérojonctions. Ainsi, les $\varphi_{n}$ étaient des arcs de sinusoïde et les $E_{n}=(\hbar \pi n)^{2} /\left(2 m^{*} a^{2}\right), n=1,2, \ldots$, où $a$ était la largeur du puits. Pour une structure réelle telle que celle de la figure 1 , le problème est plus compliqué et nous avons été amenés à élaborer un programme spécial de résolution auto-consistante de l'équation de Schrödinger et de l'équation de Poisson. Nous

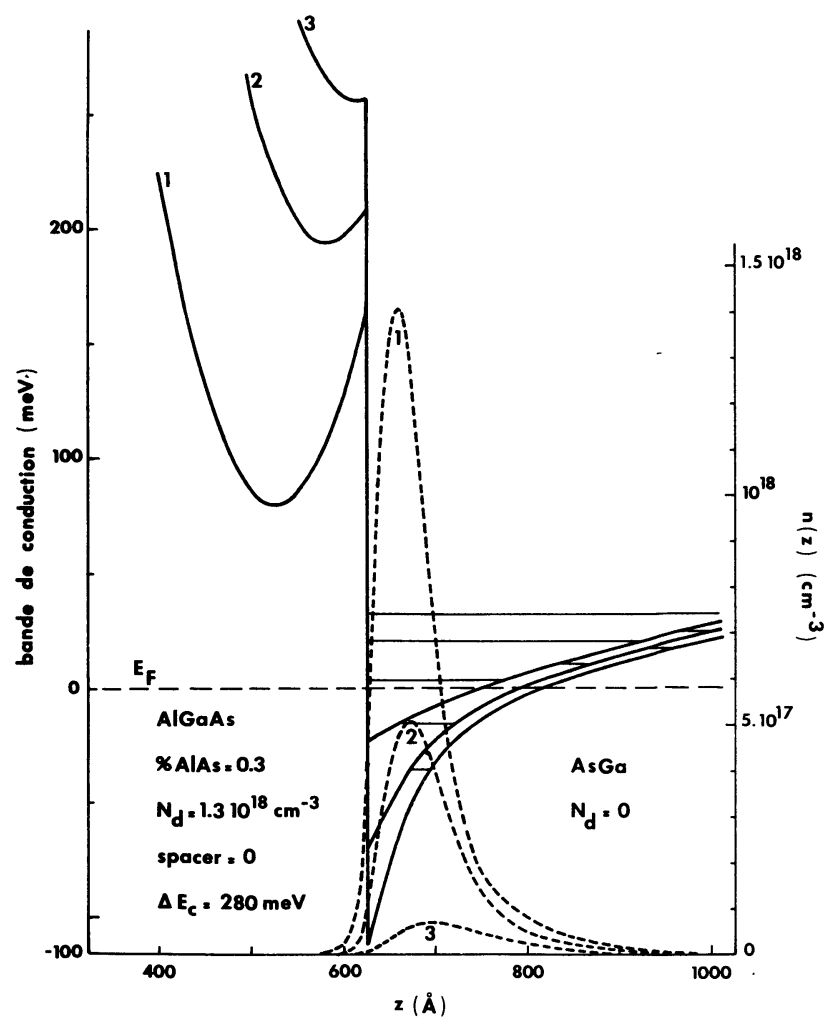

Fig. 3. - Allure du potentiel (bande de conduction) à l'hétérojonction (trait plein) et densité d'électrons (trait interrompu) dans la direction de quantification pour trois tensions de grille $\left.V_{\mathrm{g}}: 1\right) V_{\mathrm{g}}=-1,6 \mathrm{~V}, n_{\mathrm{s}}=1,15 \times 10^{12}$ $\mathrm{cm}^{-2}$; 2) $V_{\mathrm{g}}=-2,3 \mathrm{~V}, \quad n_{\mathrm{s}}=5 \times 10^{11} \mathrm{~cm}^{-2}$; 3) $V_{\mathrm{g}}$ $=-2,7 \mathrm{~V}, n_{\mathrm{s}}=9 \times 10^{10} \mathrm{~cm}^{-2}$. La température est de $77 \mathrm{~K}$. La barrière de Schottky $\phi_{\mathrm{B}}$ est supposée égale à 1 volt.

[Potential energy (full lines) and electron density (dashed lines) along the quantized $z$ direction for three different gate bias voltages.] 

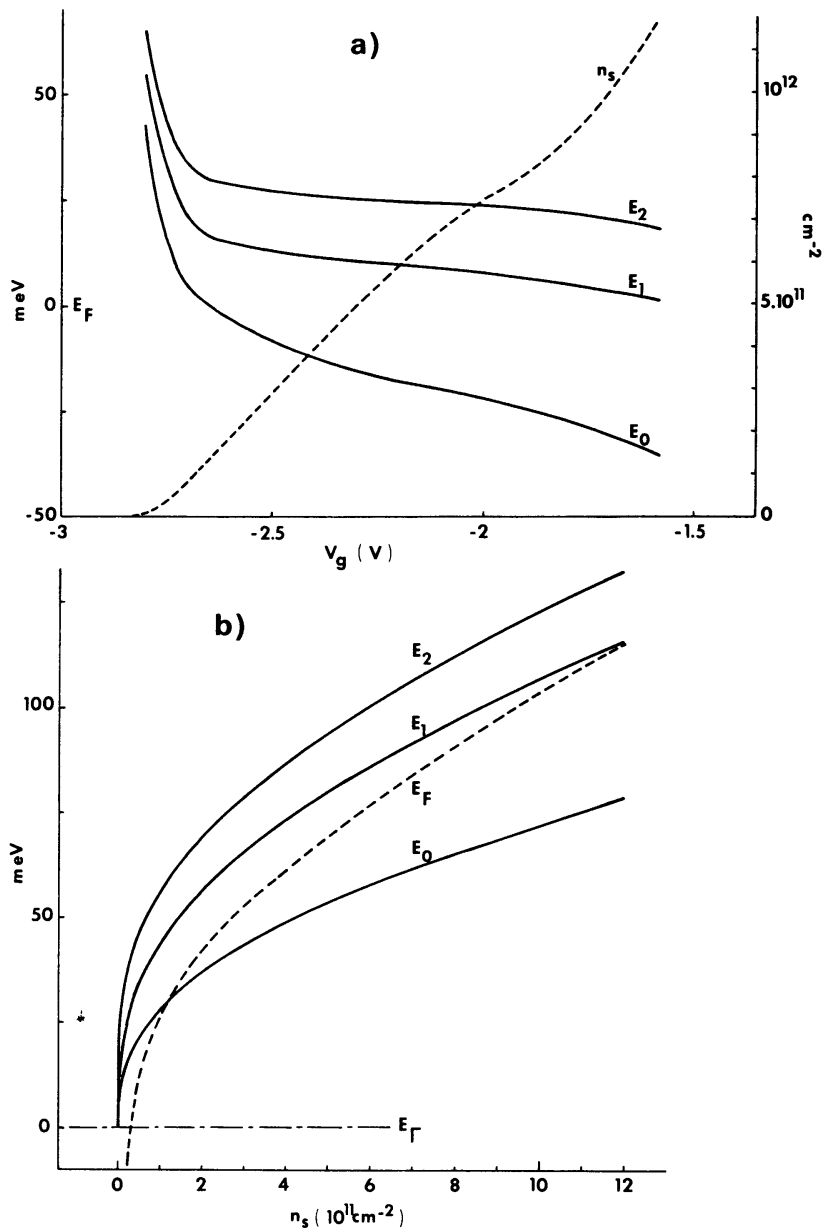

Fig. 4. - Evolution de $n_{\mathrm{s}}$ et des trois premières énergies de sous-bandes avec $V_{g}$, pour la structure de la figure 3 . Quand $n_{\mathrm{s}}$ augmente les énergies tendent à se séparer. Le cadre du bas montre les mêmes données représentées en fonction de $n_{\mathrm{s}}$ dans les mêmes conditions. Quand $n_{\mathrm{s}}$ tend vers zéro, les $E_{i}$ tendent toutes vers zéro et le niveau de Fermi rentre dans la bande interdite. Un fait remarquable est que les $E_{i}$ et $E_{\mathrm{F}}$ ainsi représentées constituent des données universelles pourvu que $\Delta E_{\mathrm{c}}$ soit plus grand que $0,2 \mathrm{~V}(x>0,2)$ et la température la même, quelles que soient par ailleurs les caractéristiques du composant. Ce fait peut être très utile pour la conception de composants optimisés en CAO.

[Top shows the variations of $n_{\mathrm{s}}$ and the first three energy subbands $E_{i}$ with $V_{\mathrm{g}}$, in the structure depicted in figure 3. Bottom, the same $E_{i}$, and $E_{\mathrm{F}}$ as functions of $n_{\mathrm{s}}$ at $T=77 \mathrm{~K}$.]

illustrons ce point avec les figures 3 et 4 . Les caractéristiques de la structure calculée sont celles indiquées dans la figure 3 . Nous y avons représenté l'évolution de la bande de conduction et de la densité d'électrons avec $z$ dans trois cas de polarisation de la grille. La figure 4a montre l'évolution des énergies de sous-bandes avec $V_{\mathrm{g}}$, ainsi que la densité surfacique $n_{\mathrm{s}}$. La figure $4 \mathrm{~b}$ montre l'évolution des mêmes énergies en fonction de $n_{\mathrm{s}}$, prenant comme référence le fond de la vallée $\Gamma$. Rappelons simplement que la densité $n_{\mathrm{s}}$ (à l'équilibre thermique) se déduit de la position des sous-bandes par rapport au niveau de Fermi [7] par :

$n_{\mathrm{s}}=\sum_{i} n_{\mathrm{s}_{i}}=\frac{m^{*} k_{\mathrm{B}} T}{\pi \hbar^{2}} \sum_{i} \ln \left[1+\exp \left(\frac{E_{\mathrm{F}}-E_{i}}{k_{\mathrm{B}} T}\right)\right]$

et la densité volumique des électrons par :

$$
n(z)=\sum_{i} n_{\mathrm{s}_{i}}\left|\varphi_{i}(z)\right|^{2}
$$

Une fois que la structure de bande du système est connue, on peut se tourner vers le problème posé par les collisions avec les défauts du cristal.

2.3 LES PROBABILITÉS DE COLlision. - Nous regardons successivement les différents cas qui interviendront tour à tour au hasard durant la simulation : celui d'un système à trois dimensions, celui d'un système à deux dimensions, enfin celui qui concerne le couplage entre les deux systèmes.

2.3.1 Cas tridimensionnel. - Il constitue l'aspect le plus connu de ce type de travail. Le modèle utilisé est celui présenté par Littlejohn et al. [8] et déjà utilisé dans notre laboratoire depuis de nombreuses années [9]. Les interactions qui sont prises en compte sont celles du schéma de bande classique de AsGa (arrangement $\Gamma$-L-X) où interviennent en plus des impuretés du matériau les phonons optiques polaires, les phonons acoustiques et acoustiques piézoélectriques (quasi élastiques), les transferts intervallés assistés par phonons... L'ensemble des paramètres mis en jeu est détaillé dans $[8,9]$.

\subsubsection{Cas bidimensionnel. - A présent l'électron} est représenté par la fonction d'onde (2) et non plus par une onde plane. A la base du calcul des probabilités de collision figure la règle d'or de Fermi valable dans tous les cas: soit $S_{3}\left(\mathbf{k}, \mathbf{k}^{\prime}\right) \mathrm{d}^{3} k^{\prime}$ la probabilité qu'un porteur dans l'état $\mathbf{k}$ subisse une collision l'amenant dans l'état $\mathbf{k}^{\prime}$ à $\mathrm{d}^{3} k^{\prime}$ près, on a (à trois dimensions) :

$$
\begin{aligned}
S_{3}\left(\mathbf{k}, \mathbf{k}^{\prime}\right) & =\frac{2 \pi}{\hbar}\left|M_{3}\left(\mathbf{k}, \mathbf{k}^{\prime}\right)\right|^{2} \delta\left(E(\mathbf{k})-E\left(\mathbf{k}^{\prime}\right) \pm \hbar \omega\right), \ldots \\
M_{3}\left(\mathbf{k}, \mathbf{k}^{\prime}\right) & =\left\langle\psi_{\mathbf{k}^{\prime}}(\mathbf{r})|V(\mathbf{r})| \psi_{\mathbf{k}}(\mathbf{r})\right\rangle .
\end{aligned}
$$

où $V(\mathbf{r})$ est le potentiel perturbateur responsable de la collision et $\hbar \omega$ l'énergie échangée au cours de celle-ci. 
Dans le cas à deux dimensions, au lieu de (5), on a :

$$
\begin{aligned}
& S_{2}^{m, n}\left(\mathbf{k}_{\|}, \mathbf{k}_{\mid}^{\prime}\right)=\frac{2 \pi}{\hbar}\left|M_{2}^{m, n}\left(\mathbf{k}_{\|}, \mathbf{k}_{\|}^{\prime}\right)\right|^{2} \delta\left(E\left(\mathbf{k}_{\|}\right)-E\left(\mathbf{k}_{\mid}^{\prime}\right)+E_{m}-E_{n} \pm \hbar \omega\right), \\
& M_{2}^{m, n}\left(\mathbf{k}_{\|}, \mathbf{k}_{\mid}^{\prime}\right)=\left\langle\psi_{n, \mathbf{k}_{\mid}}\left(\mathbf{r}_{\|}, z\right)\left|V\left(\mathbf{r}_{\|}, z\right)\right| \psi_{m, \mathbf{k}_{\mid}}\left(\mathbf{r}_{\|}, z\right)\right\rangle .
\end{aligned}
$$

qui représente la densité de probabilité qu'un porteur dans l'état $\mathbf{k}_{\mathbf{y}}$ de la sous-bande $m$ subisse une collision l'amenant dans l'état $k_{\mid}^{\prime}$ de la sous-bande $n$. De (2), (5) et (6), on tire la propriété suivante :

$$
\left|M_{2}^{m, n}\left(\mathbf{k}_{\|}, \mathbf{k}_{\|}^{\prime}\right)\right|^{2}=\int\left|M_{3}\left(\mathbf{k}, \mathbf{k}^{\prime}\right)\right|^{2}\left|I_{m n}\left(q_{z}\right)\right|^{2} \mathrm{~d} q_{z}, \ldots
$$

avec

$$
I_{m n}\left(q_{z}\right)=\int \varphi_{m}(z) \varphi_{n}(z) \exp \left(i q_{z}\right) \mathrm{d} z, \ldots
$$

où $q_{z}$ est la composante de $\mathbf{q}=\mathbf{k}-\mathbf{k}^{\prime}$ le long de la direction $z$.

Les potentiels $V$ apparaissant dans (5) et (6) sont identiques et égaux aux différents potentiels connus à trois dimensions pour chaque type d'interaction. En toute rigueur, le potentiel figurant dans (6) devrait être défini pour représenter celui qui existe à l'interface. En fait,dans l'ignorance de ce dernier on utilise les potentiels du matériau en volume, donc définis à trois dimensions, ce qui se justifie par le bon accord de maille entre les deux matériaux qui assure la continuité de la structure cristalline à travers l'hétérojonction. Ceci peut ne pas être le cas des hétérojonctions contraintes. de

En pratique dans la simulation, nous avons besoin

$$
\lambda_{2}^{m n}\left(E_{\|}\right)=\lambda_{2}^{m n}\left(\mathbf{k}_{\|}\right)=\int S_{2}^{m n}\left(\mathbf{k}_{\|}, \mathbf{k}_{\|}^{\prime}\right) d^{2} k_{\|}^{\prime},
$$

qui représente la probabilité par unité de temps qu'un porteur situé dans l'état $\mathbf{k}_{\boldsymbol{\|}}$ de la sous-bande $m$ effectue une transition vers un état quelconque de la sous-bande $n$. Le nouvel état $\mathbf{k}$ / après la collision est entièrement spécifié par les principes de conservation de l'énergie et du moment totaux, de façon analogue à ce qui se fait habituellement à trois dimensions [10-12]. De cette manière, nous traitons toutes les interactions autres que celles dues aux impuretés ionisées, c'est-à-dire les collisions avec les phonons optiques polaires, les phonons acoustiques et piézoélectriques de la vallée $\Gamma$ à deux dimensions.

A titre d'illustration, la figure 5 montre l'évolution des probabilités de collision sur phonons optiques polaires quand on inclut trois sous-bandes. Il faut voir que la difficulté essentielle et incontournable de ce genre de modèle est que les probabilités de collision changent suivant la configuration de la structure étudiée en accord avec les modifications induites au niveau des fonctions d'onde et des sousbandes par la polarisation de grille, par exemple.

Concernant l'interaction sur impuretés ionisées, nous avons utilisé la méthode de Lee [13] que nous

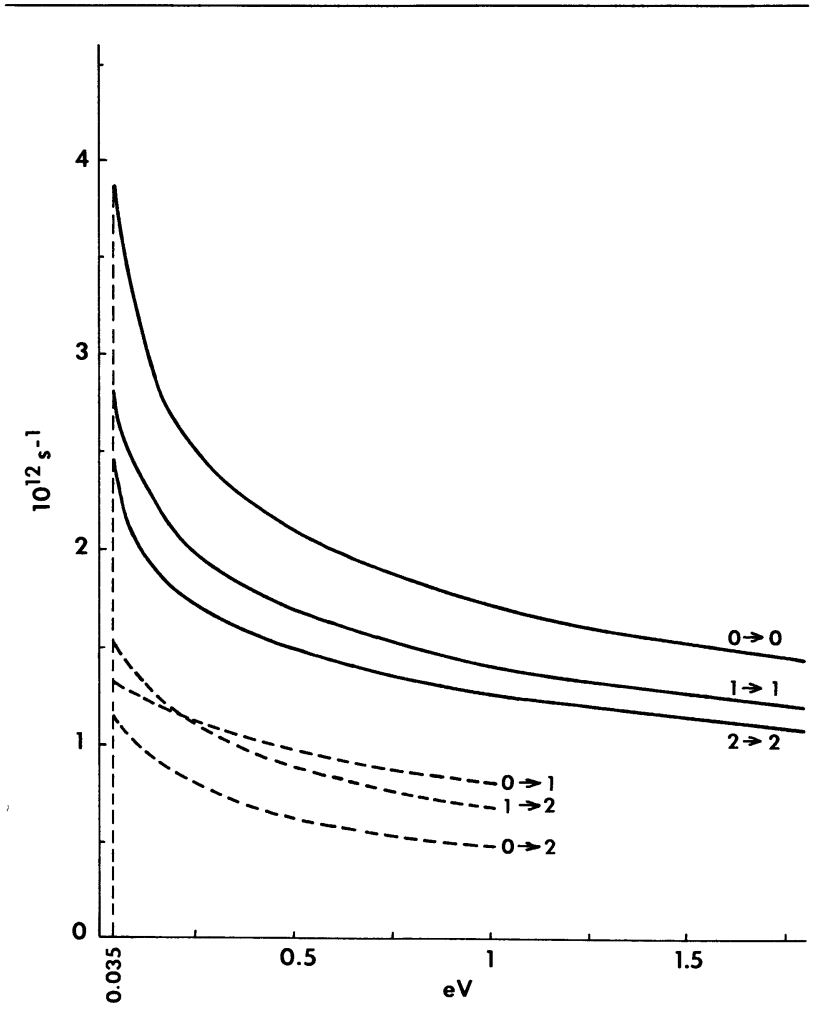

Fig. 5. - Probabilités de collisions par émission de phonons optiques polaires. A trois sous-bandes sont associées six quantités de ce type (plus six autres pour l'absorption, non représentée ici). En trait plein : collisions intra sous-bandes; en trait interrompu : collisions inter sous-bandes. Cas de la structure de la figure 3 pour $n_{\mathrm{s}}=1,5 \times 10^{11} \mathrm{~cm}^{-2}$, et $T=77 \mathrm{~K}$.

[Scattering rates with polar-optical phonon emission, for three subbands. Full lines: intra-subband collisions; dashed lines : intersubband collision. Case of figure 3 with $n_{\mathrm{s}}=1.5 \times 10^{11} \mathrm{~cm}^{-2}$ and $T=77 \mathrm{~K}$.]

avons généralisée au cas d'un nombre quelconque de sous-bandes. A ce niveau, on doit discerner les collisions sur « impuretés éloignées » (les ions de la couche $\mathrm{AlGaAs}$ fortement dopée) de celles dues aux 
« impuretés locales » (les ions résiduels de la couche AsGa non dopée). La figure 6 illustre la probabilité de collision due aux «impuretés éloignées » et ses variations avec l'ordre de la sous-bande où les porteurs se situent. Dans la mesure où les électrons des sous-bandes élevées peuvent se grouper plus loin de l'interface, donc des impuretés, que ceux des sous-bandes inférieures, la probabilité de collision associée diminue, toutes choses égales, quand l'indice de sous-bande augmente.

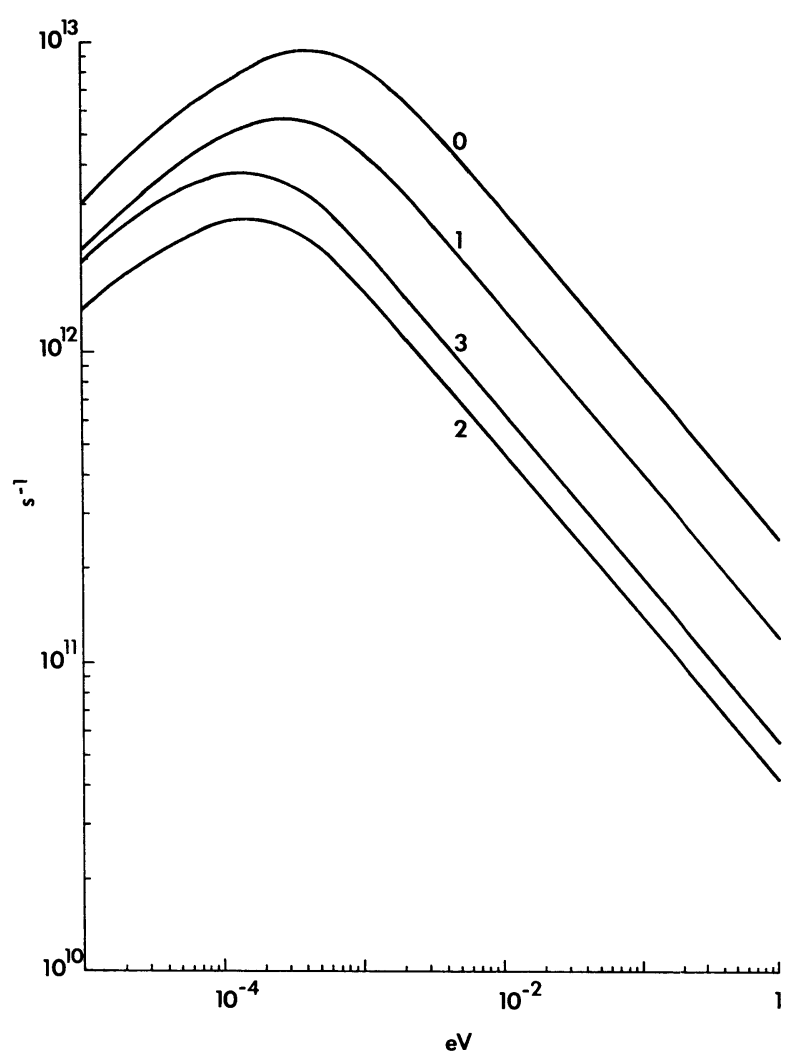

Fig. 6. - Probabilités de collisions par les «impuretés éloignées » et évolution de celles-ci avec l'énergie et l'indice de sous-bandes. Cas de la structure de la figure 3 pour $n_{\mathrm{s}}=1,5 \times 10^{11} \mathrm{~cm}^{-2}$ et $77 \mathrm{~K}$. La courbe $\mathrm{n}^{\circ} 3$ montre ce que devient la probabilité associée à la sous-bande 1 quand on introduit un «spacer » de $75 \AA \AA$.

[Scattering rates with « remote » impurities as functions of energy and subband index. Case of figure 3 with $n_{\mathrm{s}}=$ $1.5 \times 10^{11} \mathrm{~cm}^{-2}$ and $T=77 \mathrm{~K}$. Curve $n^{\circ} 3$ shows the same as for subband 1 when a spacer of $75 \AA$ is introduced.]

La prise en compte de l'effet d'écran sur un potentiel d'interaction nécessite une étude soigneuse de la fonction diélectrique du système électronique envisagé puisque l'élément de matrice écranté est obtenu par le rapport de l'élément de matrice non écranté par la fonction diélectrique. Cette dernière a été évaluée exactement par la théorie de la réponse linéaire [14], connaissant la structure de sous-bande et les fonctions d'onde électroniques dans le puits de potentiel pour être non pas intégrée directement dans la simulation, ce qui est prohibitif, mais pour servir de base à des approximations correctes. Ainsi pour chaque couple de sous-bandes $(m, n)$ on peut approximer avec une précision satisfaisante un élément de la fonction diélectrique par la forme générale $1+q_{\mathrm{s}}\left(n_{\mathrm{s}}, T\right) / q$, pour les collisions élastiques, où $q_{\mathrm{s}}$ dépend aussi des incides $m$ et $n$. C'est en ce sens que la théorie de Lee et al. a été généralisée. Il faut noter cependant que l'approche utilisée ici est tout à fait générale et applicable à tous les types de potentiels d'interaction induisant des collisions élastiques. Pour les collisions inélastiques, le problème est un peu plus compliqué dans la mesure où la fonction diélectrique dependrait aussi de la fréquence. Néanmoins si cette dernière est connue, l'approche utilisée ici pourra être appliquée sans difficultés supplémentaires.

Nous n'envisageons pas ici la possible limitation de mobilité due au type et à la qualité de l'interface. On suppose celle-ci parfaitement abrupte et dénuée de défauts, en égard aux techniques de croisance habituellement utilisée dans ce domaine et au bon accord de maille obtenu à l'interface GaAlAs sur GaAs. Donc tous les effets dûs aux collisions avec les défauts d'interface (charges fixes, dislocations, ...) ne sont pas pris en compte. Ceci ne constitue pas vraiment une limitation du présent modèle dans la mesure où ces divers effets ont déja été modélisés dans les études du transport aux interfaces $\mathrm{Si}_{-} \mathrm{SiO}_{2}$ des canaux des transistors MOS et ces modèles sont en pratique applicables aux hétérojonctions de matériaux III-V. Il est toutefois probable que des défauts de structure d'interface jouent un rôle important dans les hétérojonctions contraintes.

La mise en œuvre du modèle ainsi constitué ne présente pas de difficultés particulières. La différence essentielle avec le cas à trois dimensions est que l'on a un seul angle de déviation (mouvement plan) au lieu de deux. A l'angle manquant se substitue l'indice de sous-bande qui est devenu un variable aléatoire dans le problème. De plus, à cause du nombre de sous-bandes considérées, il y a multiplication du nombre de probabilités de collisions à évaluer et tester en cours de simulation. Par là, nous faisons référence aux deux indices $m$ et $n$ présents dans l'expression (9).

2.3.3 Couplage entre un système à deux dimensions et un système à trois dimensions. - A tous points de vue, cet aspect constitue la pierre de touche du modèle. Tant que le champ électrique accélérateur est faible, le système électronique reste au voisinage de l'équilibre ; les électrons restant dans la vallée $\Gamma$ à deux dimensions, leur mouvement est strictement bidimensionnel. Ce qui caractérise les électrons dans cet état est que leur abscisse $z$ ne peut prendre que des valeurs proches de celles occupées par les lobes de la fonction d'onde électronique de la sous-bande 
où ils se trouvent. Là où la fonction d'onde est faible, un électron n'a que peu de chances de se trouver. Il est donc naturel de supposer qu'un électron accéléré et échauffé par le champ électrique et ainsi parvenu en vallée satellite ne peut revenir dans la vallée $\Gamma$ à deux dimensions que si sa position selon $z$ le situe au-dessus d'un lobe d'une des fonctions d'onde existant dans le puits. En d'autres termes, l'électron doit être relativement près de l'hétérojonction. Si ce n'est pas le cas, une transition d'une vallée satellite vers une vallée $\Gamma$ à trois dimensions reste toujours possible. En fait en vallée satellite, l'électron connaît un mouvement de diffusion à trois dimensions identique à celui qui existe dans le matériau en volume, ce qui peut résulter en un échappement de l'électron vers le substrat ou vers la couche AlGaAs. Mais la courbure de la bande de conduction dans ce cas (équivalente à un champ dans la direction $z$ ) repoussera plus ou moins fortement cet électron vers l'interface et vers le puits. Il en résulte que hors de l'équilibre en champ accélérateur fort - le système d'électrons sera constitué pour parties de porteurs strictement bidimensionnels (dans la vallée $\Gamma$ ), de porteurs strictement tridimensionnels répartis entre les deux vallées satellites et la vallée $\Gamma$ à trois dimensíons. Dans de telles conditions nous avons choisi de caractériser les probabilités de collisions intervallées de la façon suivante. Nous disons que la probabilité de collision par unité de temps qu'un électron à deux dimensions transfère vers une vallée satellite dépend de son énergie et de sa position $z$, et vaut (pour un porteur de la sousbande $n$ ) :

$$
\begin{aligned}
\lambda_{\Gamma_{2 \mathrm{~d}} \rightarrow S_{3 \mathrm{~d}}}^{n} & \left(E_{\|}, z\right)= \\
& =\lambda_{\Gamma_{3 \mathrm{~d}} \rightarrow S_{3 \mathrm{~d}}}\left(E_{n}+E_{\|}\right) \cdot 3 b_{n}\left|\varphi_{n}(z)\right|^{2},
\end{aligned}
$$

où $\lambda_{\Gamma_{3 d} \rightarrow S_{3 d}}(E)$ sont les expressions habituelles pour le cas à trois dimensions $[9,10],\left|\varphi_{n}(z)\right|^{2}$ est la densité de probabilité de présence de l'électron en $z$, et $b_{n}$ est la largeur effective de la sous-bande $n$ définie par :

$$
b_{n}=\frac{1}{2}\left[\int_{-\infty}^{+\infty} \varphi_{n}^{4}(z) \mathrm{d} z\right]^{-1}
$$

$b_{n}$ représente en fait l'encombrement spatial de la couche électronique sur la $n$-ième sous-bande dans la direction $z$. Enfin, dans (10) $E=E_{n}+E_{\|}$est l'énergie totale de la particule à l'instant de la collision. Quand le porteur est à deux dimensions, $z$ peut être obtenue par une méthode de Von Neumann [15] appliquée à $\left|\varphi_{n}(z)\right|^{2}$. Pour la transition inverse, nous utilisons ( $E$ étant l'énergie cinétique à trois dimensions) :

$$
\begin{aligned}
& \lambda_{S_{3 \mathrm{~d}} \rightarrow \Gamma_{2 \mathrm{~d}}}^{n}(E, z)= \\
& \quad=\lambda_{S_{3 \mathrm{~d}} \rightarrow \Gamma_{3 \mathrm{~d}}}\left(E-E_{n}\right) \cdot 3 b_{n}\left|\varphi_{n}(z)\right|^{2} .
\end{aligned}
$$

Dans ce dernier cas, $z$ est connue puisque l'état de départ est à trois dimensions, et l'abcisse $z$ est donnée par la simulation elle-même. On voit que, ce faisant, on introduit une simple modulation dans l'espace des probabilités de collisions ordinaires à trois dimensions telles qu'on peut les trouver dans $[8,9]$. Le facteur $3 b_{n}$ apparaissant dans (10) et (12) assure la dimensionnalité de la probabilité de collision et a été choisi ainsi car ce facteur représente la largeur physique du puits de potentiel au niveau de l'énergie de sous-bande considérée. $3 b_{n}$ représente exactement cette largeur dans le cas d'un puits rectangulaire très profond.

Quand l'électron est assez loin dans le substrat, les $\varphi_{n}(z)$ sont amorties et tendent vers zéro, les transitions représentées par (10) et (12) ne sont plus possibles. Par contre les électrons restent toujours en communication avec la vallée $\Gamma$ à trois dimensions. En accord avec (10) et (12) nous écrivons que les probabilités de transfert associées sont :

$$
\begin{gathered}
\lambda_{\Gamma \rightarrow S}=\lambda_{\Gamma_{3 \mathrm{~d}} \rightarrow S_{3 \mathrm{~d}}} . F(z) ; \\
\lambda_{S \rightarrow \Gamma}=\lambda_{S_{3 \mathrm{~d}} \rightarrow \Gamma_{3 \mathrm{~d}}} \cdot F(z) \\
F(z)=1-\sum_{n} 3 b_{n}\left|\varphi_{n}(z)\right|^{2},
\end{gathered}
$$

qui sont les valeurs complémentaires des expressions (10) et (12) sommées sur le nombre total de sousbandes considérées dans le puits. Un exemple de fonction $F(z)$ est montré sur la figure 7. Là où $F(z) \simeq 1$, le transfert en vallée $\Gamma$ ne peut être que purement tridimensionnel. Au voisinage du puits de potentiel, $F(z)$ présente une diminution très mar-

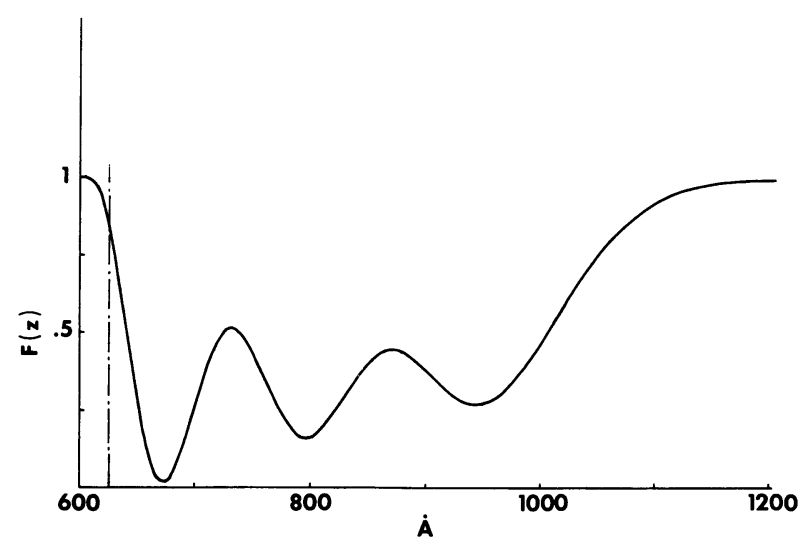

Fig. 7. - Dans cette fonction $F(z)$, trois sous-bandes sont inclues. Cas de la figure 3 pour $n_{\mathrm{s}}=9 \times 10^{10} \mathrm{~cm}^{-2}$, ̀̀ $77 \mathrm{~K}$. En deça de $600 \AA$ et au-delà de $1200 \AA$, le mouvement des électrons sera purement tridimensionnel. Ailleurs, il sera mitigé avec une prépondérance au mouvement à deux dimensions aux alentours de $680 \AA$. L'hétérojonction se situe à $625 \AA$.

[Three subbands are included in this $F(z)$ (see text). Case of figure 3 with $n_{\mathrm{s}}=9 \times 10^{10} \mathrm{~cm}^{-2}$ and $T=77 \mathrm{~K}$.] 
quée qui montre que le transfert vers la vallée $\Gamma$ à deux dimensions est pratiquement seul autorisé.

\section{Résultats.}

Pour les personnes qui travaillent à la conception de composants, certains paramètres sont nécessaires pour permettre une optimisation des conditions de fonctionnement. Au premier chef figure la mobilité électronique et ses variations avec les polarisations appliquées au composant. Viennent ensuite la vitesse de dérive en fonction du champ électrique et le coefficient de diffusion.

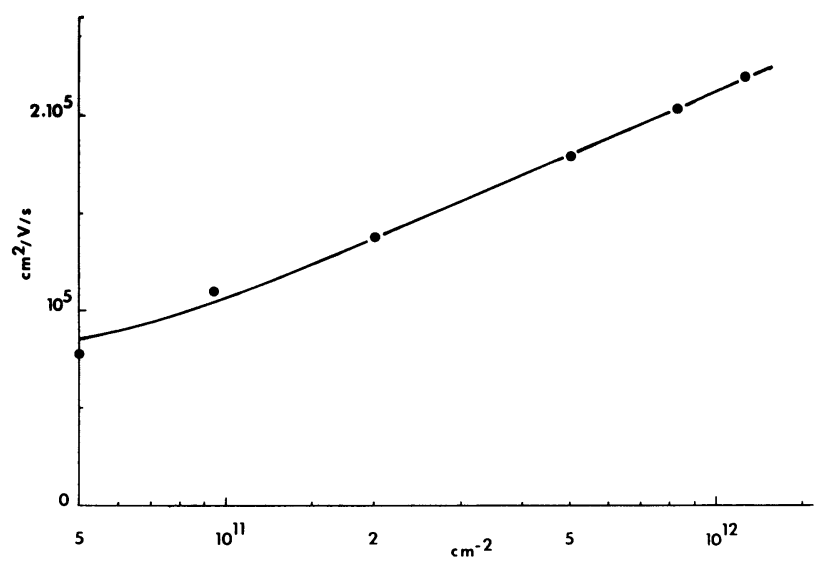

Fig. 8. - Cas de la structure de la figure 3 à $77 \mathrm{~K}$. Variation de la mobilité bas champ (calculée à $20 \mathrm{~V} / \mathrm{cm}$ ) avec $n_{\mathrm{s}}$. L'augmentation importante à $n_{\mathrm{s}}$ élevé traduit l'effet du phénomène d'écrantage des électrons du canal sur le potentiel coulombien dû aux «impuretés éloignées ».

[Low field $(20 \mathrm{~V} / \mathrm{cm})$ mobility as a function of $n_{\mathrm{s}}$. Case of figure 3 at $77 \mathrm{~K}$.]

La figure 8 montre comment varie la mobilité électronique avec la densité surfacique $n_{\mathrm{s}}$ dans la structure décrite en figure 3 . On note que la mobilité augmente quand $n_{\mathrm{s}}$ croît. Deux phénomènes contribuent à faire varier cette mobilité quand $n_{\mathrm{s}}$ change. D'une part, l'effet des largeurs effectives $b_{n}$ des sous-bandes $[15,16]$ telles que définies par l'équation (11) : ces quantités interviennent dans le calcul des $\lambda_{2}^{m, n}\left(E_{\|}\right)$; quand $n_{\mathrm{s}}$ augmente, les $b_{n}$ diminuent et $\lambda_{2}^{m, n}\left(E_{\|}\right)$augmente ce qui résulte en une augmentation du nombre de collisions donc en une diminution de la mobilité (ceci est vrai pour toutes les collisions, inclues celles sur les impuretés ionisées). D'autre part, il existe l'effet de l'écrantage du potentiel coulombien dû aux impuretés ionisées par les électrons. Cet écrantage devient de plus en plus actif quand $n_{\mathrm{s}}$ augmente. Il en résulte une diminution du taux de collision sur les impuretés et une augmentation de la mobilité. On note que dans tous les cas considérés jusqu'à présent le second phénomène prend largement le pas sur le premier ce qui conduit à une augmentation de la mobilité quand $n_{\mathrm{s}}$ augmente.

La figure 9 présente l'évolution de la vitesse de dérive électronique (et de l'énergie moyenne des porteurs) en fonction du champ accélérateur appliqué suivant la direction $x$. On note que l'allure est celle habituelle connue par AsGa, toutefois la vitesse pic de l'ordre de $2,8 \times 10^{7} \mathrm{~cm} / \mathrm{s}$ est obtenue à des champs plus faibles (ici de l'ordre de $1200 \mathrm{~V} / \mathrm{cm}$ ) que ceux obtenus dans le cas à trois dimensions (de l'ordre de $3000 \mathrm{~V} / \mathrm{cm}$ ). Ce phénomène peut se corréler à une diminution de la hauteur effective de la barrière séparant la vallée $\Gamma$ des vallées satellites. Le passage des électrons en vallées satellites est d'autant plus aisé que cette hauteur de barrière est faible. Dans le cas présent, cette barrière est abaissée d'une quantité d'énergie égale à l'énergie de la sousbande où se situe l'électron par rapport au fond de la vallée $\Gamma$ comme il a été montré dans la figure $4 \mathrm{~b}$. Néanmoins la précision du calcul ne nous a pas permis jusqu'à présent de savoir si la position des sous-bandes (donc la valeur de $n_{\mathrm{s}}$ ) a un effet sensible sur la vitesse pic et sur le champ de seuil. En réalité, cette partie de la courbe est de loin la plus difficile à déterminer avec précision. Au-delà du champ de seuil, toutefois, l'allure de la courbe reste très similaire à ce que l'on obtient dans le cas de AsGa peu dopé à trois dimensions.

Les résultats obtenus ici sont assez similaires à ceux obtenus par d'autres auteurs [17-19]. Une comparaison systématique de nos résultats avec ces derniers est toutefois pratiquement impossible dans la mesure où les structures simulées sont différentes dans chaque cas et conduisent donc à des résultats différents. D'une façon générale, néanmoins, on trouve qu'une mobilité différentielle négative dans la courbe de vitesse de dérive en fonction du champ électrique se manifeste à des champs nettement moins élevés que dans le cas purement tridimensionnel [20]. Un aspect intéressant de la référence [18] est la prise en compte de la rétroaction du phénomène d'électrons chauds sur la structure des sousbandes du système lui-même. En effet, du fait de la présence du champ électrique accélérateur, il y a modification de la population des sous-bandes, ce qui dans l'hypothèse d'une statistique de Fermi considérée comme encore valable dans ce cas permet de spécifier la nouvelle position des sous-bandes par l'intermédiaire de la relation (3). La résolution de l'équation de Schrödinger tenant compte de ces nouveaux niveaux d'énergies propres permet d'obtenir la nouvelle allure des fonctions d'onde, de la charge électronique et par suite la nouvelle forme du puits de potentiel. L'ensemble des équations est résolu régulièrement au cours de la simulation. On note toutefois que les résultats acquis, bien que plus rigoureux en principe, sont assez peu différents de 


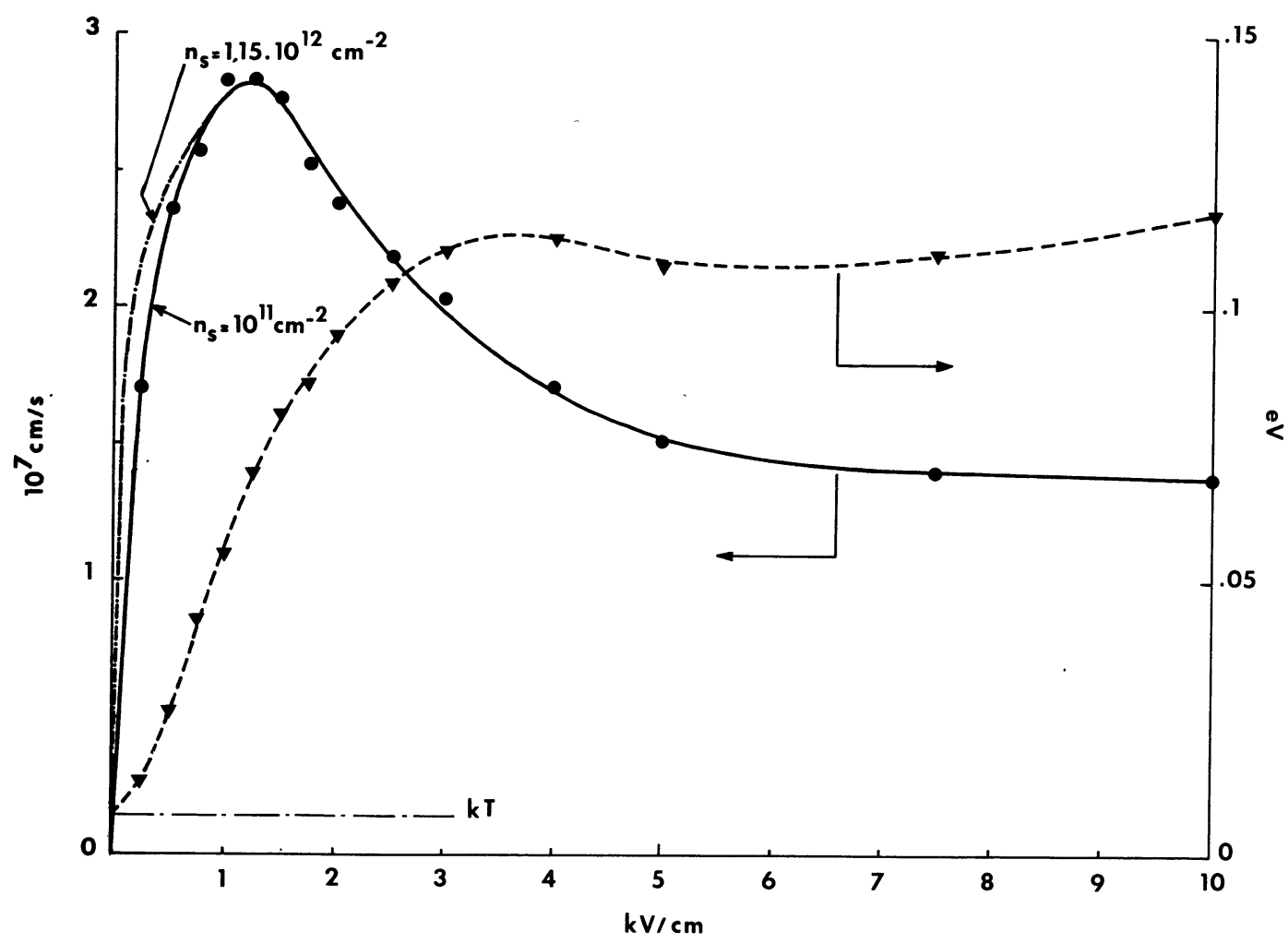

Fig. 9. - Structure de la figure 3 à $77 \mathrm{~K}$, à $n_{\mathrm{s}}=1,15 \times 10^{12} \mathrm{~cm}^{-2}$ et $1 \times 10^{11} \mathrm{~cm}^{-2}$. Variations de la vitesse de dérive (trait plein) et de l'énergie moyenne (trait interrompu) avec le champ électrique. Une vitesse pic de $2,8 \times 10^{7} \mathrm{~cm} / \mathrm{s}$ est obtenue vers un champ de seuil de $1200 \mathrm{~V} / \mathrm{cm}$. La diminution rapide de la vitesse au-dessus de ce champ correspond au transfert de plus en plus dense des électrons vers les vallées satellites où le mouvement devient tridimensionnel. Vitesse pic et champ de seuil restent apparemment peu sensibles à la valeur de $n_{\mathrm{s}}$. Seul le régime ohmique y est sensible. De même, l'énergie moyenne reste quasiment insensible à la valeur de $n_{\mathrm{s}}$ dans tout le domaine de champ électrique étudié. Nous l'avons représentée en une seule courbe pour les deux valeurs de $n_{\mathrm{s}}$ étudiées dans la figure. Les flèches rectangulaires montrent l'échelle à lire pour chaque quantité calculée.

[Drift velocity (full and dash-dotted lines) and mean electron energy (dashed line) as functions of the electric field, for two values of $n_{\mathrm{s}}$ at $77 \mathrm{~K}$. Peak-velocity and threshold field look to be rather insensitive to the change of $n_{\mathrm{s}}$; this is not the case of the ohmic regime however. But the mean energy is quite the same whatever the value of $n_{\mathrm{s}}$. (The rectangular arrows show the scale to be read).]

ceux obtenus en supposant une structure qui reste en permanence à l'équilibre [21].

En relation directe avec la figure 9, nous avons tracé sur la figure 10 l'évolution des différentes populations des vallées en fonction du champ électrique. On note que dès que le champ augmente, la sous-bande fondamentale (0) commence à se vider dans la sous-bande (1) puis dans la sous-bande (2). Aux alentours du champ de seuil, vers $1000-1500$ $\mathrm{V} / \mathrm{cm}$, la population de ces deux dernières a considérablement augmenté alors que les vallées satellites restent encore relativement peu peuplées. Ces dernières commencent à se peupler au-delà de $2000 \mathrm{~V} / \mathrm{cm}$; dans le même temps les trois sousbandes voient leurs populations diminuer régulièrement. On note toutefois que celles-ci restent relativement élevées même en champ fort. Il existe donc un transfert assez important des vallées satellites vers les vallées $\Gamma$ à deux dimensions. On note aussi que le transfert des vallées satellites vers la vallée $\Gamma$ à trois dimensions reste tout à fait négligeable.

Dans la figure 11, nous montrons l'évolution spatiale de la charge d'espace des électrons à deux dimensions dans le système. La rétroaction des électrons chauds sur la structure des sous-bandes n'étant pas prise en compte ici, la charge d'espace reste confinée aux dimensions géométriques du puits telles que calculées au départ (Fig. 3). La prise en compte de cette rétroaction aurait pour résultat de permettre à la charge d'espace de s'étaler beaucoup plus vers le substrat (à champ élevé). A cela correspondrait un élargissement du puits de potentiel, graduellement jusqu'à disparition du puits et du caractère bidimensionnel des électrons.

Nous abordons maintenant le dernier point de cette présentation: il concerne le phénomène de diffusion. Ce phénomène qui est la source du bruit de diffusion dans les composants trouve son impor- 


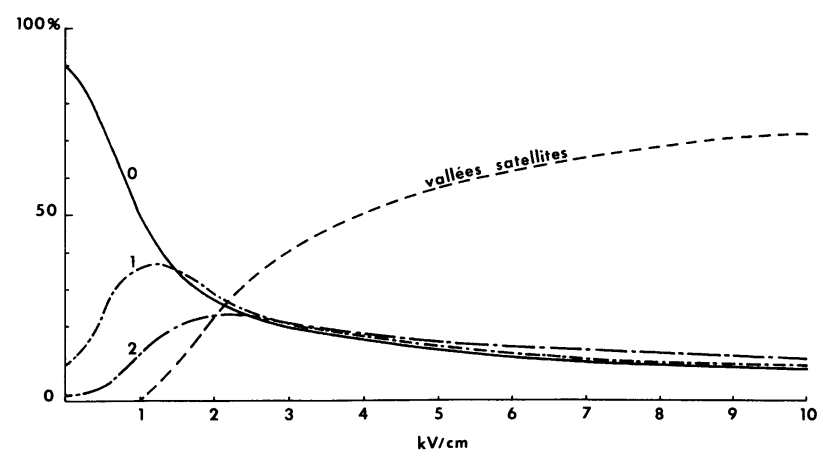

Fig. 10. - Evolution en pourcent du peuplement des trois sous-bandes et des vallées satellites. Cas de la figure 9 à $n_{\mathrm{s}}=1,15 \times 10^{12} \mathrm{~cm}^{-2}$. Aux alentours du champ de seuil, la sous-bande 0 s'est dépeuplée en faveur des sous-bandes 1 et 2. Au-delà, l'ensemble transfère ses électrons en vallées satellites. En champ fort les trois sous-bandes restent à peu près également peuplées $(10 \%)$. La courbure de bande de conduction des vallées satellites vers l'hétérojonction maintient, même en champ fort, un peuplement relativement important du puits quantique.

[Population (in \%) of subbands and satellite valleys as functions of the electric field (case of figure 9 at $n_{\mathrm{s}}=$ $\left.1.15 \times 10^{12} \mathrm{~cm}^{-2}\right)$.]

tance dans le fait qu'il est le phénomène le plus fondamental qui limite leur fonctionnement. Aux fréquences supérieures à $10 \mathrm{GHz}$, par exemple, les bruits de grenaille ou de génération-recombinaison sont totalement éliminés; reste seulement le plus souvent le bruit de diffusion qui existe toujours du fait que le composant et les porteurs sont à une température non nulle. Ce bruit de diffusion est dû aux fluctuations microscopiques de la vitesse des porteurs à l'intérieur du système. Ce bruit se caractérise en termes de coefficients de diffusion et nous renvoyons le lecteur aux références [ 22 et 23] pour une étude plus approfondie du phénomène en question. Nous nous contenterons présentement de rappeler les relations fondamentales dans la suite de cet exposé.

Nous calculons le coefficient de diffusion par les mêmes moyens que ceux utilisés en général pour le cas des systèmes tridimensionnels [22]. Nous nous restreignons ici toutefois à l'étude d'un système strictement bidimensionnel c'est-à-dire, pour la structure étudiée jusqu'à présent dans cet article, aux champs électriques plus petits que $600 \mathrm{~V} / \mathrm{cm}$. Nous laissons donc de côté pour le moment le problème de bruit de diffusion lié au transfert vers les vallées satellites. De façon conventionnelle nous définissons les coefficients de diffusion de deux façons différentes : soit par la caractérisation temporelle de l'étalement d'un paquet de porteurs (1000 dans la pratique) qui initialement se trouvant au même point du plan $(x, y)$ se disperse dans ce plan au cours du temps; soit par le calcul de la fonction

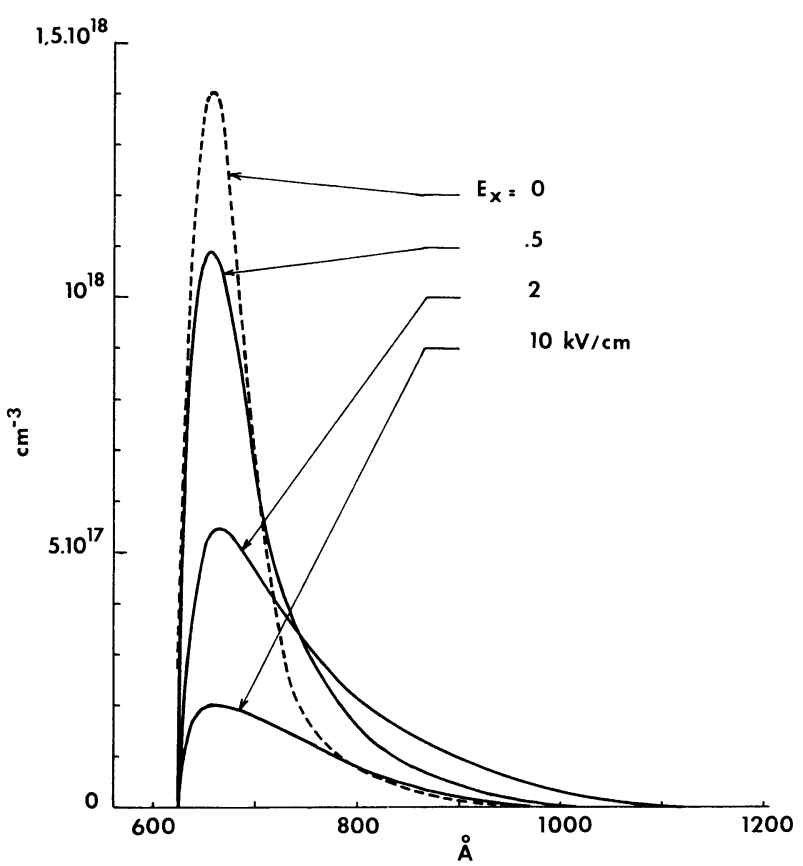

Fig. 11. - Même cas que la figure 10. Evolution spatiale, avec $z$, de la densité des électrons à deux dimensions. On note que l'étalement de la charge d'espace n'est pas fortement modifié par les conditions de champ électrique ; seule l'aire sous la courbe (voir Fig. 10) diminue quand le champ augmente. Ceci est dû à la rigidité des fonctions d'onde du puits qui, dans le présent modèle, restent celles de l'équilibre (champ faible) même en champ fort. Un modèle plus rigoureux, recalculant les fonctions d'onde d'après la température effective des électrons dans la structure toute entière, montrerait également un étalement de la charge d'espace à deux dimensions vers le substrat, éventuellement jusqu'à disparition du puits et de l'aspect bidimensionnel des électrons.

[Space variation of the two-dimensional gas density as it changes with the driving electric field strength. Case of figure 10.]

de corrélation des fluctuations des vitesses des porteurs en fonction du temps.

Dans la première méthode, la position des porteurs $x(t)$ et $y(t)$ sont calculés en cours de simulation et la variance de ces déplacements est calculée par :

$$
\begin{aligned}
& \operatorname{var}_{x}(t)=\overline{(x(t)-\bar{x}(t))^{2}}, \\
& \operatorname{var}_{y}(t)=\overline{(y(t)-\bar{y}(t))^{2}},
\end{aligned}
$$

où les moyennes représentées par une barre sont effectuées à chaque instant sur l'ensemble des électrons simulés. Les coefficients de diffusion associés sont alors définis par :

$$
\begin{aligned}
& D_{x}=\lim \frac{1}{2 t} \operatorname{var}_{x}(t), \quad t \rightarrow \infty, \\
& D_{y}=\lim \frac{1}{2 t} \operatorname{var}_{y}(t), \quad t \rightarrow \infty .
\end{aligned}
$$


Aux temps $t$ suffisamment grands, on doit observer que les variances évoluent linéairement avec le temps. Un résultat de ce type est montré sur la figure 12. Cette figure montre que la linéarité est assez bien obtenue dans le domaine temporel étudié (0 à $350 \mathrm{ps}$ ). Elle montre aussi que cette linéarité peut déjà être observée à des temps nettement plus courts (elle apparaît en fait dès la cinquantaine de picosecondes). La figure 12 montre aussi que la variance de déplacement est plus faible dans la direction $x$ (qui est celle du champ électrique) comparée à ce qu'elle est dans la direction perpendiculaire $y$. Il en résultera que le coefficient $D_{x}$ sera

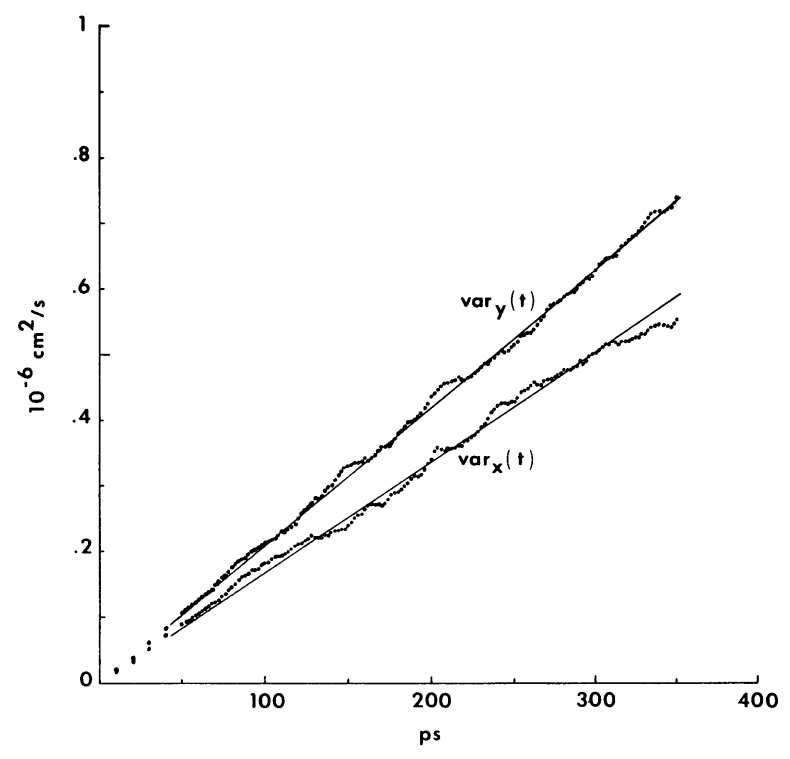

Fig. 12. - Même structure que la figure 3, mais à $n_{\mathrm{s}}=1 \times 10^{11} \mathrm{~cm}^{-2}$ et $200 \mathrm{~V} / \mathrm{cm}$. Cette figure montre l'évolution temporelle des variances de déplacements des électrons selon $x$ et $y$. La linéarité est assez bien obtenue sur le domaine temporel considéré. Durant leur mouvement les 1000 électrons simulés restent bidimensionnels.

[Mean-square $x$ and $y$ displacements of a bunch of electron as a function of time. Structure of figure 3 at $n_{\mathrm{s}}=$ $10^{11} \mathrm{~cm}^{-2}$ and $E=200 \mathrm{~V} / \mathrm{cm}$ parallel to $x .1000$ electrons are simulated.]

plus petit que le coefficient $D_{y}$. Ceci est conforme à ce qui est habituellement obtenu à trois dimensions où le coefficient de diffusion dans la direction du champ électrique est toujours plus faible que dans la direction perpendiculaire [22, 23]. Toujours d'après la figure 12 , on peut noter qu'en moyenne, au bout de $350 \mathrm{ps}$, un électron peut s'être éloigné de près de $10 \mu \mathrm{m}$ de son point de départ. En regard, le mouvement de diffusion dans la direction $z$ où les états des porteurs sont quantifiés et de ce fait, limité à $100-200 \AA$, est proprement négligeable; à cela correspond un coefficient de diffusion $D_{z}$ qui est virtuellement nul. Il n'en sera plus de même dans le cas du champ fort lorsqu'il autorisera les porteurs à transférer en vallée satellite où le mouvement selon $z$ redevient libre.

La seconde méthode de calcul du coefficient de diffusion utilisant la fonction de corrélation des fluctuations de vitesses est illustrée par la figure 13. $\mathrm{Si}$ au cours de la simulation on connaît les valeurs des composantes $v_{x}$ et $v_{y}$ de la vitesse d'un électron, on peut définir des fonctions de corrélation de fluctuation de ces quantités par :

$\phi_{x}(t)=\lim _{T \rightarrow \infty} \frac{1}{T} \int_{0}^{T}\left(v_{x}(t+u)-\bar{v}_{x}\right)\left(v_{x}(u)-\bar{v}_{x}\right) \mathrm{d} u$

$\phi_{y}(t)=\lim _{T \rightarrow \infty} \frac{1}{T} \int_{0}^{T}\left(v_{y}(t+u)-\bar{v}_{y}\right)\left(v_{y}(u)-\bar{v}_{y}\right) \mathrm{d} u$.

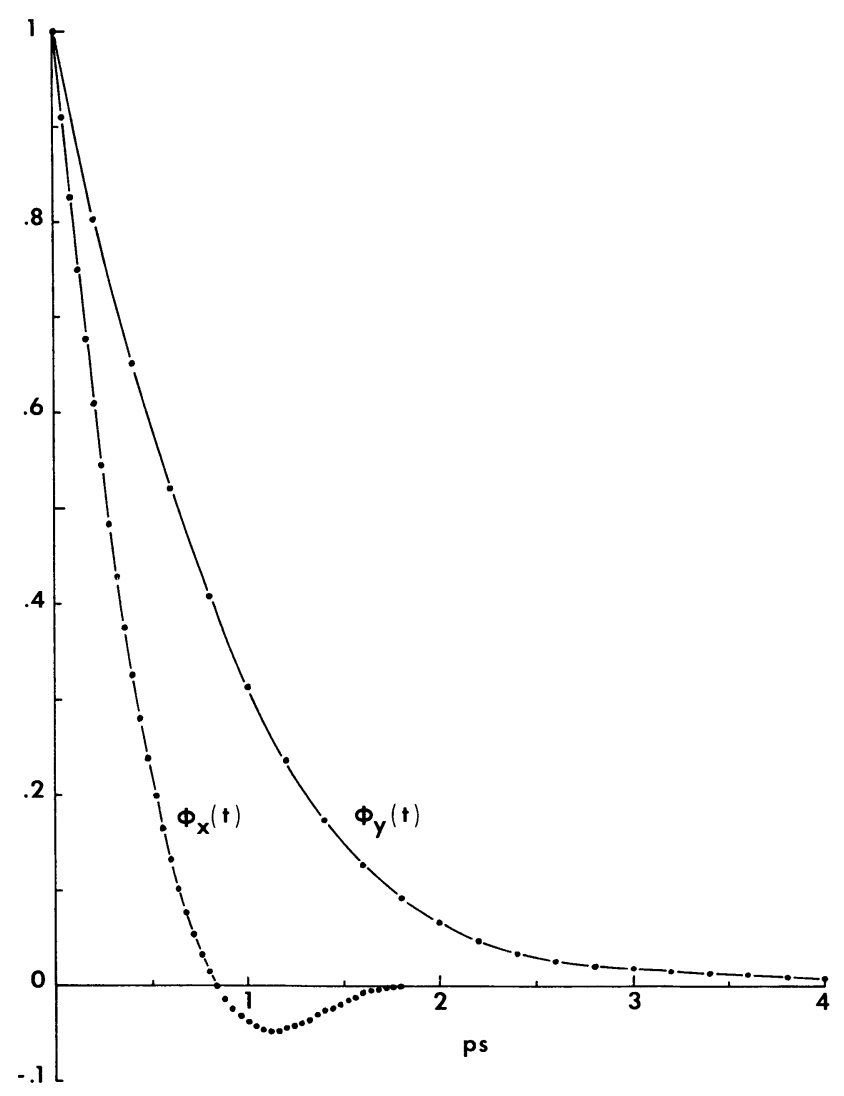

Fig. 13. - Même structure que la figure 3, avec $n=1 \times$ $10^{11} \mathrm{~cm}^{-2}, T=77 \mathrm{~K}$ et à $400 \mathrm{~V} / \mathrm{cm}$. Fonctions de corrélation des vitesses suivant $x$ et $y$ réduites à 1 à $t=0$. La fonction de corrélation suivant le champ appliqué selon $x$ présente une décroissance plus rapide suivie d'un minimum vers des valeurs négatives. Il en résulte un temps de corrélation plus court qui a pour effet de donner un coefficient de diffusion plus petit dans cette direction que dans le sens perpendiculaire au champ électrique.

[ $x$ and $y$ velocity fluctuation correlation function reduced to 1 at $t=0$, obtained in structure of figure 3 at $n_{\mathrm{s}}=10^{11} \mathrm{~cm}^{-2}$ and $E=400 \mathrm{~V} / \mathrm{cm}$ applied along $x$.] 
Si le système est en régime stationnaire on définit les coefficients de diffusion par:

$$
\begin{aligned}
& D_{x}=\int_{0}^{\infty} \phi_{x}(t) \mathrm{d} t, \\
& D_{y}=\int_{0}^{\infty} \phi_{y}(t) \mathrm{d} t .
\end{aligned}
$$

L'équivalence numérique et formelle des définitions (16) et (18) des coefficients de diffusion, ainsi que les conditions requises pour qu'elle se réalise, ont été montrées de détail dans $[9,15,22,23]$.

La fonction de corrélation de $v_{x}$ est caractérisée essentiellement par une décroissance plus rapide que celle observée pour la fonction de corrélation de $v_{y}$ et aussi par un passage vers les valeurs négatives. Au contraire, la fonction de corrélation de $v_{y}$ reste toujours positive. Ce comportement particulier avait déjà été noté pour l'AsGa à trois dimensions [22] et expliqué par le transfert des électrons en vallées satellites. Dans le cas de la figure 13, le transfert en vallées satellites n'existe pas encore, par contre le transfert des électrons entre les sous-bandes existe réellement et pourrait expliquer le phénomène. Il résulte de la valeur des fonctions de corrélation que

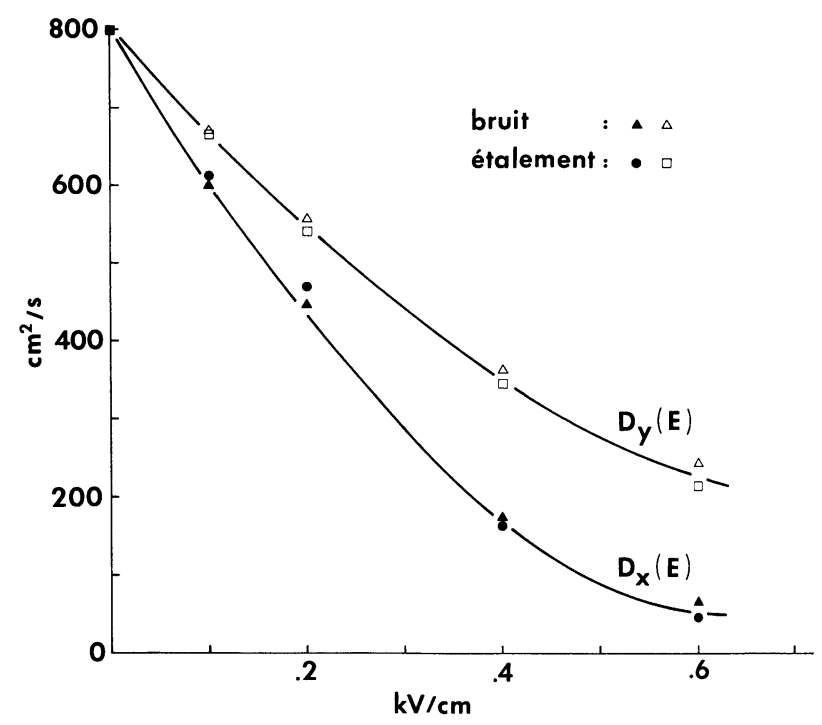

Fig. 14. - Evolution des coefficients de diffusion suivant $x$ (direction du champ électrique accélérateur) et suivant $y$ en fonction de ce champ. On note la décroissance rapide de ces coefficients quand le champ augmente, tant que le mouvement des électrons reste bidimensionnel.

[Diffusion coefficients along the field $(D x)$ and transverse $(D y)$ as functions of the electric field. Structure of figure 3 at $n_{\mathrm{s}}=10^{11} \mathrm{~cm}^{-2}$ and $T=77 \mathrm{~K}$.]
$D_{x}$ doit être nettement inférieur à $D_{y}$. L'ensemble des résultats acquis jusqu'à maintenant sont résumés dans la figure 14, où nous avons représenté l'évolution des coefficients de diffusion avec le champ électrique. On a toujours noté une bonne concordance entre les coefficients calculés par les deux méthodes. On a noté ainsi que ces coefficients diminuent très nettement lorsque le champ augmente. Il y a tout lieu de penser qu'aux champs plus élevés, le transfert en vallées satellites donnera lieu à une augmentation rapide de ceux-ci au fur et à mesure que les électrons deviendront tridimensionnels durant ce transfert. Cet aspect intéressant de la question n'a toutefois pas encore été abordé, mais le sera dans un proche avenir.

\section{Conclusion.}

Dans cette présentation, nous avons reporté essentiellement des résultats obtenus à relativement basse température, $77 \mathrm{~K}$. Les paramètres calculés sont en effet plus sensibles au champ électrique à ces températures qu'à la température ambiante. Les études que nous avons effectuées à $300 \mathrm{~K}$ ont montré que les résultats obtenus se rapprochent beaucoup de ce que l'on connaît pour AsGa en volume et qu'en première approximation il peut être légitime de les utiliser pour la simulation de composants à hétérojonctions. A basse température, notre opinion sur ce point de vue est beaucoup plus réservée. Il est certain de plus qu'il existe encore à l'heure actuelle un manque de données expérimentale dans ce domaine. Des mesures visant à déterminer la vitesse de dérive en champ fort ou les coefficients de diffusion, quelle que soit la technique utilisée, ne sont ni aisément réalisables ni facilement interprétables. En regard de cela, les modélisations basées sur la dynamique microscopiques trouvent donc leur pleine utilité. Une difficulté réside toutefois dans l'interaction des structures simulées et des polarisations de celles-ci sur la dynamique microscopique elle-même. Ceci rend difficilement comparables les résultats acquis indépendamment par les auteurs et oblige à considérer chaque structure ou chaque type de composant comme un cas particulier. Les modèles physiques de conception de composants assistés par ordinateur (nous entendons par là, les modèles n'utilisant pas de schémas électriques équivalents) trouvent leur utilité s'ils sont suffisamment simples pour donner des résultats probants en peu de temps calculs. Ils doivent donc s'appuyer sur des descriptions de paramètres physiques qui ne peuvent être obtenues que par des modèles plus élaborés dont celui présenté ici est un exemple. 


\section{Bibliographie}

[1] Le HEMT (High Electron Mobility Transistor) est également connu sous les noms de TEGFET (Two-dimensional Electron Gas Field Effect Transistor), de MODFET (Modulation Doping FET), et de SDHT (Selectively Doped Heterojunction Transistor).

[2] Dingle, R., IEEE Trans. ED-31 (1984) 1662.

[3] Hiyamizu, S. et al., Jpn J. Appl. Phys. 20 (1981) L245.

[4] Delescluse, P. et al., Electron. Lett. 17 (1981) 44.

[5] LiNH, N. T., NATO Advanced Studies Institute (San Miniato, Italie) Juillet 1983.

[6] Heiblum, M. et al., Appl. Phys. Lett. 44 (1984) 1064.

[7] En pratique, la résolution de l'équation de Schrödinger donne les valeurs des énergies propres $E_{n}$, dont on tire le niveau de Fermi pour un $n_{s}$ donné d'avance par la méthode de Newton appliquée à l'équation (3). L'équation de Poisson appliquée à l'équation (4) permet par double intégration d'obtenir la valeur de $V_{\mathrm{g}}$ correspondante. L'évaluation de la charge ionique $N_{D}^{+}$ dans AlGaAs a été faite dans la plupart des cas en admettant une énergie d'ionisation apparente dépendant de la fraction molaire de AlAs dans le matériau. Voir à ce sujet par exemple :

Chand, N. et al., Phys. Rev. B 30 (1984) 4481 ; Vinter, B., Appl. Phys. Lett. 44 (1984) 307 ou CAPPY, A., Thèse de Doctorat d'Etat, Université de Lille I (décembre 1986).

[8] Littlejohn, M. A. et al., J. Appl. Phys. 48 (1977) 4587.

[9] KASZYNSKI, A., Thèse de Docteur Ingénieur, Université de Lille I (juillet 1979).

[10] Fawcett, W. et al., J. Phys. Chem. Solids 31 (1970) 1963.

[11] Price, P. J., Ann. Phys. (N.Y.) 133 (1981) 217.

[12] Price, P. J., J. Vac. Sci. Technol. 19 (1981) 599.

[13] LeE, J. et al., J. Appl. Phys. 54 (1983) 6995.

[14] Siggia, E. D. et KwoK, P. C., Phys. Rev. B 2 (1970) 1024.

[15] Price, P. J., Semiconductors and Semimetals R. K. Willardson et A. C. Beer, Ed. 14 (Academic Press, New-York) p. 264.

[16] ZimmermanN, J. et al., Physica 129B (1985) 385.

[17] Al-Mudares, M. et al., J. Phys. C. 19 (1986) 3179.

[18] Yoкоуама, K. et al., J. Appl. Phys. 59 (1986) 3798.

[19] MiYatsugi, K., et al., Compt. rend. S.S.D.M. Tokyo (1986).

[20] Ruch, J. G. et Fawcett, W., J. Appl. Phys. 41 (1970) 3843.

[21] Yokoyama, K. et Hess, K., Phys. Rev. B 33 (1986) 5595.

[22] Fauquembergue, R. et al., J. Appl. Phys. 51 (1980) 2065.

[23] HiLl, G. et al., J. Appl. Phys. 50 (1979) 356. 